\title{
Algebraic shifting and strongly edge decomposable complexes
}

\author{
Satoshi Murai $\|^{\dagger}$ \\ Department of Pure and Applied Mathematics, Graduate School of Information Science and Technology, Osaka \\ University, Toyonaka, Osaka, 560-0043, Japan
}

\begin{abstract}
Let $\Gamma$ be a simplicial complex with $n$ vertices, and let $\Delta(\Gamma)$ be either its exterior algebraic shifted complex or its symmetric algebraic shifted complex. If $\Gamma$ is a simplicial sphere, then it is known that (a) $\Delta(\Gamma)$ is pure and (b) $h$-vector of $\Gamma$ is symmetric. Kalai and Sarkaria conjectured that if $\Gamma$ is a simplicial sphere then its algebraic shifting also satisfies (c) $\Delta(\Gamma) \subset \Delta(C(n, d))$, where $C(n, d)$ is the boundary complex of the cyclic $d$-polytope with $n$ vertices. We show this conjecture for strongly edge decomposable spheres introduced by Nevo. We also show that any shifted simplicial complex satisfying (a), (b) and (c) is the algebraic shifted complex of some simplicial sphere.
\end{abstract}

2000 Mathematics Subject Classification. 13F55

Keywords: Algebraic shifting, Simplicial spheres, The strong Lefschetz property, Squeezed spheres

\section{Introduction}

Algebraic shifting, which was introduced by , is a map which associates with each simplicial complex $\Gamma$ another simplicial complex $\Delta(\Gamma)$ having a simple structure, called shifted. There are two main variants of algebraic shifting, called exterior algebraic shifting $\Delta^{e}(-)$ and symmetric algebraic shifting $\Delta^{s}(-)$ (see $\S 2$ for the detail). The simplicial complex $\Delta^{e}(\Gamma)$ (resp. $\Delta^{s}(\Gamma)$ ) is called the exterior (resp. symmetric) algebraic shifted complex of $\Gamma$. The main application of algebraic shifting is the study of face vectors of simplicial complexes. Indeed algebraic shifting has been giving several remarkable results on this ground (see Björner and Kalai (1989)). On the other hand, one of the major open problem in the theory of face vectors of simplicial complexes is the characterization of face vectors of simplicial spheres (that is, simplicial complexes whose geometric realization is homeomorphic to a sphere). Let $\Delta(-)$ be either $\Delta^{e}(-)$ or $\Delta^{s}(-)$. If $\Gamma$ is a $(d-1)$-dimensional simplicial sphere then it is known that $\Delta(\Gamma)$ satisfies the following properties (see $\S 1$ for the definition of the $h$-vector of simplicial complexes).

(a) $\Delta(\Gamma)$ is pure, that is, all facets of $\Delta(\Gamma)$ have the same cardinality;

(b) the $h$-vector of $\Delta(\Gamma)$ is symmetric, that is, $h_{i}(\Delta(\Gamma))=h_{d-i}(\Delta(\Gamma))$ for $i=0,1, \ldots, d$.

\footnotetext{
${ }^{\dagger}$ The author is supported by JSPS Research Fellowships for Young Scientists

1365-8050 @ 2008 Discrete Mathematics and Theoretical Computer Science (DMTCS), Nancy, France
} 
To characterize face vectors of simplicial spheres by using algebraic shifting, Kalai and Sarkaria suggested the following conjecture.

Conjecture 1 (Kalai, Sarkaria) Let $C(n, d)$ be the boundary complex of a cyclic d-polytope with $n$ vertices. If $\Gamma$ is a $(d-1)$-dimensional simplicial sphere with $n$ vertices then

$$
\Delta(\Gamma) \subset \Delta^{s}(C(n, d)) .
$$

An important fact on this conjecture is that if Conjecture 1 is true (for either exterior shifting or symmetric shifting) then it yields the characterization of face vectors of simplicial spheres (see Kalai (1991, 2002)). Moreover, Kalai conjectured that condition (a), (b) and (c) characterize algebraic shifted complexes of simplicial spheres.

In this paper, we give two results on Conjecture 1 . First, we show that Conjecture 1 is true for simplicial spheres satisfying a certain combinatorial property, called strongly edge decomposable. (Note that, for symmetric algebraic shifting, this result was essentially proved in Babson and Nevo (2008).) Second, we show that for any $(d-1)$-dimensional pure shifted complex $\Sigma$ with $n$ vertices satisfying $\Sigma \subset \Delta^{s}(C(n, d))$ and $h_{i}(\Sigma)=h_{d-i}(\Sigma)$ for $i=0,1, \ldots, d$, there exists a simplicial sphere $\Gamma$ such that $\Delta^{e}(\Gamma)=\Delta^{s}(\Gamma)=$ $\Sigma$. Thus, if Conjecture 1 is true, this result gives the sufficiency of the characterization of algebraic shifted complexes of simplicial spheres.

\section{Strongly edge decomposable simplicial complexes}

In this section we study the strongly edge decomposable property introduced by $\operatorname{Nevo}(2006)$. Let $\Gamma$ be a simplicial complex on $[n]=\{1,2, \ldots, n\}$. Thus $\Gamma$ is a collection of subsets of $[n]$ satisfying that (i) $\{i\} \in \Gamma$ for all $i \in[n]$ and (ii) if $F \in \Gamma$ and $G \subset F$ then $G \in \Gamma$. An element $F$ of $\Gamma$ is called a face of $\Gamma$ and maximal faces of $\Gamma$ under inclusion are called facets of $\Gamma$. A simplicial complex is said to be pure if all its facets have the same cardinality. Let $f_{k}(\Gamma)$ be the number of faces $F \in \Gamma$ with $|F|=k+1$, where $|F|$ is the cardinality of $F$. The dimension of $\Gamma$ is $\operatorname{dim} \Gamma=\max \left\{k: f_{k}(\Gamma) \neq 0\right\}$. The vector $f(\Gamma)=\left(f_{0}(\Gamma), f_{1}(\Gamma), \ldots, f_{d-1}(\Gamma)\right)$ is called the $f$-vector of $\Gamma$, where $d=\operatorname{dim} \Gamma+1$.

First, we define strongly edge decomposable complexes. Let $\Gamma$ be a simplicial complex on $[n]$. The link of $\Gamma$ with respect to $F \subset[n]$ is the simplicial complex

$$
\operatorname{lk}_{\Gamma}(F)=\{G \subset[n] \backslash F: G \cup F \in \Gamma\} .
$$

To simplify, we write $\mathrm{lk}_{\Gamma}(v)=\mathrm{lk}_{\Gamma}(\{v\})$ and $\mathrm{lk}_{\Gamma}(i j)=\mathrm{lk}_{\Gamma}(\{i, j\})$. Let $1 \leq i<j \leq n$ be integers. The contraction $\mathcal{C}_{\Gamma}(i j)$ of $\Gamma$ with respect to $\{i, j\}$ is the simplicial complex on $[n] \backslash\{i\}$ which is obtained from $\Gamma$ by identifying the vertices $i$ and $j$. In other words,

$$
\mathcal{C}_{\Gamma}(i j)=\{F \in \Gamma: i \notin F\} \cup\{(F \backslash\{i\}) \cup\{j\}: i \in F \in \Gamma\} .
$$

We say that $\Gamma$ satisfies the Link condition with respect to $\{i, j\}$ if

$$
\mathrm{lk}_{\Gamma}(i) \cap \mathrm{lk}_{\Gamma}(j)=\mathrm{lk}_{\Gamma}(i j) .
$$

Definition 1.1 The boundary complex of simplexes and $\{\emptyset\}$ are strongly edge decomposable and, recursively, a pure simplicial complex $\Gamma$ is said to be strongly edge decomposable if there exists $\{i, j\} \in \Gamma$ such that $\Gamma$ satisfies the Link condition with respect to $\{i, j\}$ and both $\mathcal{C}_{\Gamma}(i j)$ and $\operatorname{lk}_{\Gamma}(i j)$ are strongly edge decomposable. 
The above definition is a natural extension of (Nevo, 2006. Definition 4.2). While Nevo assume that $\Gamma$ is a triangulated PL-manifold, we only assume that $\Gamma$ is a pure simplicial complex. Strongly edge decomposable complexes are not always a manifold, however, the class of strongly edge decomposable complexes contains a certain class of simplicial spheres. For example, 2-spheres are always strongly edge decomposable (see Nevo (2006)).

Second, we study the relation between the Link condition and shift operators. Let $\Gamma$ be a simplicial complex on $[n]$. For given integers $1 \leq i<j \leq n$ and for all $F \in \Gamma$, one defines

$$
C_{i j}(F)= \begin{cases}(F \backslash\{i\}) \cup\{j\}, & \text { if } i \in F, j \notin F \text { and }(F \backslash\{i\}) \cup\{j\} \notin \Gamma, \\ F, & \text { otherwise. }\end{cases}
$$

Let $\operatorname{Shift}_{i j}(\Gamma)=\left\{C_{i j}(F): F \in \Gamma\right\}$. It is easy to see that $\operatorname{Shift}_{i j}(\Gamma)$ is a simplicial complex satisfying $f\left(\operatorname{Shift}_{i j}(\Gamma)\right)=f(\Gamma)$ (see e.g., $($ Herzog, 2002,, 8$\left.)\right)$. The operation $\Gamma \rightarrow \operatorname{Shift}_{i j}(\Gamma)$ was introduced by Erdös et al. (1961), and played an important role in the classical extremal combinatorics of finite sets.

For a subset $F \subset[n]$ and for a simplicial complex $\Gamma$ on the vertex set $[n] \backslash F$ we write

$$
F * \Sigma=\left\{F^{\prime} \cup G: F^{\prime} \subset F \text { and } G \in \Sigma\right\}
$$

The following simple fact, which easily follows from the definition of $\operatorname{Shift}_{i j}(-)$, will play an important role in the study of algebraic shifted complexes of strongly edge decomposable complexes.

Lemma 1.2 Let $\Gamma$ be a simplicial complex on $[n]$ and let $1 \leq i<j \leq n$ be integers. Then

$$
\operatorname{Shift}_{i j}(\Gamma)=\mathcal{C}_{\Gamma}(i j) \cup\left\{\{i\} \cup F: F \in\left(\{j\} * \operatorname{lk}_{\Gamma}(i j)\right) \cup\left(\mathrm{lk}_{\Gamma}(i) \cap \mathrm{lk}_{\Gamma}(j)\right)\right\}
$$

Next, we study the $h$-vector of strongly edge decomposable complexes.

Lemma 1.3 Let $\Gamma$ be a $(d-1)$-dimensional strongly edge decomposable complex on $[n]$. Then $\operatorname{dim} \mathcal{C}_{\Gamma}(i j)=$ $d-1$ and $\operatorname{dim} \operatorname{lk}_{\Gamma}(i j)=d-3$ for any $\{i, j\} \in \Gamma$.

Proof: Since $\Gamma$ is pure, $\operatorname{dim} \operatorname{lk}_{\Gamma}(i j)=d-3$ is obvious. Suppose $\operatorname{dim} \mathcal{C}_{\Gamma}(i j)<d-1$. Then all facets of $\Gamma$ contain $\{i, j\}$. Thus $\Gamma$ is a cone (that is, $\Gamma=\{v\} * \operatorname{lk}_{\Gamma}(v)$ for some $\{v\} \in \Gamma$ ). However, if $\Gamma$ is a cone then its contraction is again a cone. This contradicts the assumption since if $\Gamma$ is strongly edge decomposable then we can obtain the boundary of a simplex by taking contractions repeatedly. Thus $\operatorname{dim} \mathcal{C}_{\Gamma}(i j)=d-1$.

Let $\Gamma$ be a $(d-1)$-dimensional simplicial complex. The $h$-vector $h(\Gamma)=\left(h_{0}(\Gamma), h_{1}(\Gamma), \ldots, h_{d}(\Gamma)\right)$ of $\Gamma$ is defined by the relations

$$
h_{i}(\Gamma)=\sum_{j=0}^{i}(-1)^{i-j}\left(\begin{array}{l}
d-j \\
d-i
\end{array}\right) f_{j-1}(\Gamma) \text { and } f_{i-1}(\Gamma)=\sum_{j=0}^{i}\left(\begin{array}{l}
d-j \\
d-i
\end{array}\right) h_{j}(\Gamma) \text {, }
$$

where we set $f_{-1}(\Gamma)=1$. If $\Gamma$ satisfies the Link condition w.r.t. $\{i, j\} \in \Gamma$ then by Lemma 1.2 we have

$$
f_{k}(\Gamma)=f_{k}\left(\mathcal{C}_{\Gamma}(i j)\right)+f_{k-1}\left(\{j\} * \operatorname{lk}_{\Gamma}(i j)\right) \text { for } k=0,1, \ldots, d-1 .
$$


Moreover, if $\operatorname{dim} \mathcal{C}_{\Gamma}(i j)=d-1$ and $\operatorname{dim} \operatorname{lk}_{\Gamma}(i j)=d-3$ then, by using the relation between $f$-vectors and $h$-vectors, we have

$$
h_{k}(\Gamma)=h_{k}\left(\mathcal{C}_{\Gamma}(i j)\right)+h_{k-1}\left(\{j\} * \mathrm{lk}_{\Gamma}(i j)\right)=h_{k}\left(\mathcal{C}_{\Gamma}(i j)\right)+h_{k-1}\left(\mathrm{lk}_{\Gamma}(i j)\right) \text { for } k=0,1, \ldots, d .
$$

Then, arguing inductively, the $h$-vector of strongly edge decomposable complexes satisfies the following conditions.

Lemma 1.4 (Nevo) Let $\Gamma$ be a $(d-1)$-dimensional strongly edge decomposable complex. Then $h_{i}(\Gamma)=$ $h_{d-i}(\Gamma)$ for $i=0,1, \ldots, d$ and $h_{0}(\Gamma) \leq h_{1}(\Gamma) \leq \cdots \leq h_{\left\lfloor\frac{d}{2}\right\rfloor}(\Gamma)$, where $\left\lfloor\frac{d}{2}\right\rfloor$ is the integer part of $\frac{d}{2}$.

Actually, the $h$-vector of strongly edge decomposable complexes satisfies a stronger condition. Indeed, it is known that strongly edge decomposable complexes have the strong Lefschetz property (see $\S 4$ ). Thus the $g$-vector of those complexes is an $M$-vector.

\section{Algebraic shifting}

In this section, we recall some basic properties of algebraic shifting. We refer the reader to Eisenbud (1995) and Herzog (2002) for the basis on Gröbner bases and generic initial ideals. Let $S=$ $K\left[x_{1}, \ldots, x_{n}\right]$ be a polynomial ring over an infinite field $K$ with each $\operatorname{deg} x_{i}=1$ and $E=\Lambda\left\langle e_{1}, \ldots, e_{n}\right\rangle$ the exterior algebra over $K$ with each $\operatorname{deg} e_{i}=1$. Let $R$ be either $S$ or $E$ and let $G L_{n}(K)$ be the general linear group with coefficients in $K$. Suppose that $G L_{n}(K)$ acts on $R$ as the group of graded $K$-algebra automorphisms. For a homogeneous ideal $I$ of $R$, we write in $(I)$ for the initial ideal of $I$ w.r.t. the reverse lexicographic order induced by $1>2>\cdots>n$. The generic initial ideal of a homogeneous ideal $I \subset R$ is $\operatorname{Gin}(I)=\operatorname{in}(\varphi(I))$ for a generic choice of $\varphi \in G L_{n}(K)$.

Exterior algebraic shifting. Let $\Gamma$ be a simplicial complex on $[n]$. For a subset $F=\left\{i_{1}, \ldots, i_{k}\right\} \subset[n]$ with $i_{1}<\cdots<i_{k}$, the element $e_{F}=e_{i_{1}} \wedge \cdots \wedge e_{i_{k}} \in E$ is called a monomial of $E$ of degree $k$. The exterior face ideal $J_{\Gamma}$ of $\Gamma$ is the ideal of $E$ generated by all monomials $e_{F} \in E$ with $F \notin \Gamma$. The exterior algebraic shifted complex $\Delta^{e}(\Gamma)$ of $\Gamma$ is defined by

$$
J_{\Delta^{e}(\Gamma)}=\operatorname{Gin}\left(J_{\Gamma}\right) .
$$

The map $\Gamma \rightarrow \Delta^{e}(\Gamma)$ is called exterior algebraic shifting. Note that $\Delta^{e}(\Gamma)$ may depend on the characteristic of the base field $K$.

Symmetric algebraic shifting. Suppose $\operatorname{char}(K)=0$. Let $\Gamma$ be a simplicial complex on $[n]$. The Stanley-Reisner ideal $I_{\Gamma}$ of $\Gamma$ is the ideal of $S$ generated by all squarefree monomials $x_{F}=\prod_{i \in F} x_{i} \in S$ with $F \notin \Gamma$. Let $\mathcal{M}$ be the set of monomials on infinitely many variables $x_{1}, x_{2}, \ldots$ The squarefree operation $\Phi: \mathcal{M} \rightarrow \mathcal{M}$ is the map defined by

$$
\Phi\left(x_{i_{1}} x_{i_{2}} x_{i_{3}} \cdots x_{i_{k}}\right)=x_{i_{1}} x_{i_{2}+1} x_{i_{3}+2} \cdots x_{i_{k}+k-1},
$$

where $i_{1} \leq i_{2} \leq \cdots \leq i_{k}$. For a monomial ideal $I$ of $S$, we write $G(I)$ for the unique minimal set of monomial generators of $I$. If $I \subset S$ is a monomial ideal satisfying $\Phi(u) \in S$ for all $u \in G(I)$, we write $\Phi(I)$ for the monomial ideal of $S$ generated by $\{\Phi(u): u \in G(I)\}$. It is known that if $I \subset S$ is a 
squarefree monomial ideal then $\Phi(u) \in S$ for all $u \in G(\operatorname{Gin}(I))$ (see e.g., (Herzog, 2002. Lemma 8.15)). The symmetric algebraic shifted complex $\Delta^{s}(\Gamma)$ of $\Gamma$ is defined by

$$
J_{\Delta^{s}(\Gamma)}=\Phi\left(\operatorname{Gin}\left(I_{\Gamma}\right)\right) .
$$

The map $\Gamma \rightarrow \Delta^{s}(\Gamma)$ is called symmetric algebraic shifting.

A simplicial complex $\Gamma$ on $[n]$ is said to be shifted if $F \in \Gamma$ and $i \in F$ imply $(F \backslash\{i\}) \cup\{j\} \in \Gamma$ for all $i<j \leq n$. Let $\Gamma$ and $\Sigma$ be simplicial complexes on $[n]$ and let $\Delta(-)$ be either $\Delta^{e}(-)$ or $\Delta^{s}(-)$. Algebraic shifting satisfies the following properties (see Herzog (2002) and Kalai (2002)).

$\left(S_{1}\right) \Delta(\Gamma)$ is shifted;

$\left(S_{2}\right)$ If $\Gamma$ is shifted then $\Delta(\Gamma)=\Gamma$;

$\left(S_{3}\right) f(\Delta(\Gamma))=f(\Gamma)$;

$\left(S_{4}\right)$ If $\Sigma \subset \Gamma$ then $\Delta(\Sigma) \subset \Delta(\Gamma)$.

Lemma 2.1 (Kalai) Let $\Delta(-)$ be either $\Delta^{e}(-)$ or $\Delta^{s}(-)$. For any simplicial complex $\Gamma$ on $[n]$, one has

$$
\Delta(\{n+1\} * \Gamma)=\{n+1\} * \Delta(\Gamma) .
$$

We say that a simplicial complex $\Gamma$ is Cohen-Macaulay if the ring $S / I_{\Gamma}$ is Cohen-Macaulay (see Stanley (1996) for further information on the Cohen-Macaulay property).

Lemma 2.2 (Kalai) If $\Gamma$ is Cohen-Macaulay over $K$ then $\Delta^{e}(\Gamma)$ (computed over $K$ ) and $\Delta^{s}(\Gamma)$ are pure. Also, a shifted complex is Cohen-Macaulay if and only if it is pure.

We refer the reader to Babson et al. (2006); Herzog (2002); Nevo (2005) for the proof of Lemmas 2.1 and 2.2. For further details on algebraic shifting, see the survey articles Herzog (2002) and Kalai (2002).

\section{Exterior shifting of strongly edge decomposable complex}

In this section, we will show that any $(d-1)$-dimensional strongly edge decomposable complex $\Gamma$ on $[n]$ satisfies $\Delta^{e}(\Gamma) \subset \Delta^{s}(C(n, d))$. We first recall the structure of $\Delta^{s}(C(n, d))$. Fix integers $n>d>0$. For integers $1 \leq i<j \leq n$, we write $[i]=\{1,2, \ldots, i\}$ and write $[i, j]=\{i, i+1, \ldots, j-1, j\}$. For $i=0,1, \ldots,\left\lfloor\frac{\bar{d}}{2}\right\rfloor$, let

$$
W_{i}(n, d)=\{([n-d+i, n] \backslash\{n-d+i\}) \cup F: F \subset[n-d+i-1],|F|=i\}
$$

and

$$
W_{d-i}(n, d)=\{([n-d+i, n] \backslash\{n-i\}) \cup F: F \subset[n-d+i-1],|F|=i\} .
$$

Let $\Delta(n, d)$ be the simplicial complex generated by $\bigcup_{i=0}^{d} W_{i}(n, d)$ and let $\Delta(n, 0)=\{\emptyset\}$. The following fact is known (see Kalai (1991) and Murai (2007a)).

Lemma 3.1 Let $n>d>0$ be integers and let $C(n, d)$ be the boundary complex of a cyclic d-polytope with $n$ vertices. Then

$$
\Delta^{e}(C(n, d))=\Delta^{s}(C(n, d))=\Delta(n, d) .
$$


To study Conjecture 1 , we use nongeneric algebraic shifting. Let $\Gamma$ be a simplicial complex on $[n]$. For any $\varphi \in G L_{n}(K)$, we write $\Delta_{\varphi}(\Gamma)$ for the simplicial complex defined by

$$
J_{\Delta_{\varphi}(\Gamma)}=\operatorname{in}\left(\varphi\left(J_{\Gamma}\right)\right) .
$$

The next fact can be proved in the same way as (Murai, 2007a. Proposition 2.4) by using (Murai 2007a. Lemma 1.5).

Lemma 3.2 Let $\Gamma$ be a $(d-1)$-dimensional simplicial complex on $[n]$. If $\Delta^{e}\left(\Delta_{\varphi}(\Gamma)\right) \subset \Delta(n, d)$ for some $\varphi \in G L_{n}(K)$ then $\Delta^{e}(\Gamma) \subset \Delta(n, d)$.

Lemma 3.2 has a nice relation to shift operators. The following fact is known (see Herzog (2002)).

Lemma 3.3 Let $1 \leq i<j \leq n$ be integers and let $\varphi_{i j} \in G L_{n}(K)$ be the graded K-algebra automorphism of $E$ induced by $\varphi_{i j}\left(e_{k}\right)=e_{k}$ for $k \neq j$ and $\varphi_{i j}\left(e_{j}\right)=e_{i}+e_{j}$. Then, for any simplicial complex $\Gamma$ on $[n]$, one has

$$
\operatorname{Shift}_{i j}(\Gamma)=\Delta_{\varphi_{i j}}(\Gamma) .
$$

We also need the following fact, which immediately follows from (Nevo, 2005, Corollary 4.4).

Lemma 3.4 Let $\Gamma$ be a simplicial complex on $\{m, m+1, \ldots, n\}$ with $1 \leq m \leq n$ and $J_{\Gamma} \subset \bigwedge\left\langle e_{m}, \ldots, e_{n}\right\rangle$ the exterior face ideal of $\Gamma$. Let $J_{\Gamma}+\left(e_{1}, \ldots, e_{m-1}\right)$ be the ideal of $E$ generated by $G\left(J_{\Gamma}\right)$ and $e_{1}, \ldots, e_{m-1}$. Then

$$
\operatorname{Gin}\left(J_{\Gamma}+\left(e_{1}, \ldots, e_{m-1}\right)\right)=\operatorname{Gin}\left(J_{\Gamma}\right)+\left(e_{1}, \ldots, e_{m-1}\right) .
$$

Next, we study the structure of $\Delta(n, d)$. Let $V \subset[n]$ and let $\Gamma$ be a simplicial complex on $V$. Consider the exterior face ideal $J_{\Gamma}$ in $\Lambda\left\langle e_{k}: k \in V\right\rangle$ and define the simplicial complex $\Delta^{e}(\Gamma)$ on $V$ by $J_{\Delta^{e}(\Gamma)}=\operatorname{Gin}\left(J_{\Gamma}\right)$. Let $C(V, d)$ be the boundary complex of a cyclic $d$-polytope with the vertex set $V$. Set $\Delta(V, d)=\Delta^{e}(C(V, d))$ for $d>0$ and $\Delta(V, 0)=\{\emptyset\}$.

Lemma 3.5 Let $n>d \geq 0$ be integers. Then $\Delta([2, n], d) \subset \Delta(n, d)$ and $\{1, n+1\} * \Delta([2, n], d) \subset$ $\Delta(n+1, d+2)$.

Proof: If $d=0$ then the statement is obvious. Suppose $d>0$. Let $F$ be a facet of $\Delta([2, n], d)$. Clearly $\Delta([2, n], d)=\left\{\left\{i_{1}, \ldots, i_{k}\right\}:\left\{i_{1}-1, \ldots, i_{k}-1\right\} \in \Delta(n-1, d)\right\}$ by Lemma 3.1. Thus there exists $0 \leq i \leq \frac{d}{2}$ and $F^{\prime} \subset[2, n-d+i-1]$ such that

$$
F=([n-d+i, n] \backslash\{n-d+i\}) \cup F^{\prime}
$$

or

$$
F=([n-d+i, n] \backslash\{n-i\}) \cup F^{\prime} .
$$

In both cases, it is clear that $F \in \Delta(n, d)$ and hence $\Delta([2, n], d) \subset \Delta(n, d)$. Also, a routine computation implies that if $F$ is an element of the form (1) then $\{1, n+1\} \cup F \in W_{i+1}(n+1, d+2)$, and if $F$ is an element of the form 22 then $\{1, n+1\} \cup F \in W_{d+2-(i+1)}(n+1, d+2)$. Thus we have $\{1, n+1\} * \Delta([2, n], d) \subset \Delta(n+1, d+2)$.

Now we will show that any $(d-1)$-dimensional strongly edge decomposable complex $\Gamma$ on $[n]$ satisfies $\Delta^{e}(\Gamma) \subset \Delta^{s}(C(n, d))$. We say that a $(d-1)$-dimensional simplicial complex $\Gamma$ on $V \subset[n]$ satisfies the shifting-theoretic upper bound relation if $\Delta^{e}(\Gamma) \subset \Delta(V, d)$. 
Proposition 3.6 Let $\Gamma$ be a $(d-1)$-dimensional simplicial complex on $[n]$ and let $1 \leq i<j \leq n$ be integers. Suppose $\operatorname{dim} \mathcal{C}_{\Gamma}(i j)=d-1$ and $\operatorname{dim} \operatorname{lk}_{\Gamma}(i) \cap \mathrm{k}_{\Gamma}(j)=d-3$. If $\mathcal{C}_{\Gamma}(i j)$ and $\operatorname{lk}_{\Gamma}(i) \cap \mathrm{k}_{\Gamma}(j)$ satisfy the shifting-theoretic upper bound relation then $\Gamma$ satisfies the shifting-theoretic upper bound relation.

Proof: By Lemmas 3.2 and 3.3, it is enough to show that $\Delta^{e}\left(\operatorname{Shift}_{i j}(\Gamma)\right) \subset \Delta(n, d)$. Let $\Gamma_{1}=\mathcal{C}_{\Gamma}(i j)$, $\Gamma_{2}=\{j\} *\left(\mathrm{lk}_{\Gamma}(i) \cap \mathrm{k}_{\Gamma}(j)\right)$ and $\Sigma=\Gamma_{1} \cup\left\{\{i\} \cup F: F \in \Gamma_{2}\right\}$. Then $\Sigma$ is a simplicial complex on $[n]$ since $\{j\} *\left(\operatorname{lk}_{\Gamma}(i) \cap \mathrm{lk}_{\Gamma}(j)\right) \subset \mathcal{C}_{\Gamma}(i j)$. Also, since $\operatorname{lk}_{\Gamma}(i) \cap \operatorname{lk}_{\Gamma}(j) \supset \operatorname{lk}_{\Gamma}(i j)$, we have $\operatorname{Shift}_{i j}(\Gamma) \subset \Sigma$ by Lemma 1.2 Thus, by $\left(S_{4}\right)$, it suffices to show that $\Delta^{e}(\Sigma) \subset \Delta(n, d)$.

Since $\Delta^{e}(\Gamma)$ is independent of the labeling of the vertices of $\Gamma$ (see Kalai (2002)), we may assume that $i=1, j=n$ and the vertex set of $\operatorname{lk}_{\Gamma}(i) \cap \mathrm{lk}_{\Gamma}(j)$ is a set of the form $[m, n-1]$ for some $m \geq 2$. Set $E^{\prime}=\bigwedge\left\langle e_{2}, \ldots, e_{n}\right\rangle$ and $\tilde{E}=\bigwedge\left\langle e_{m}, \ldots, e_{n}\right\rangle$. Let $J_{\Gamma_{1}} \subset E^{\prime}$ be the exterior face ideal of $\Gamma_{1}$ and $J_{\Gamma_{2}} \subset \tilde{E}$ the exterior face ideal of $\Gamma_{2}$. Then, since $\Sigma=\Gamma_{1} \cup\left\{\{1\} \cup F: F \in \Gamma_{2}\right\}$, we have

$$
J_{\Sigma}=J_{\Gamma_{1}} \bigoplus e_{1} \wedge\left(J_{\Gamma_{2}}+\left(e_{2}, \ldots, e_{m-1}\right)\right),
$$

where $J_{\Gamma_{2}}+\left(e_{2}, \ldots, e_{m-1}\right)$ is an ideal of $E^{\prime}$. Then there exists a $\varphi \in G L_{n-1}(K)$ which acts on $E^{\prime}$ such that $\operatorname{in}\left(\varphi\left(J_{\Gamma_{1}}\right)\right)=\operatorname{Gin}\left(J_{\Gamma_{1}}\right)$ and $\operatorname{in}\left(\varphi\left(J_{\Gamma_{2}}+\left(e_{2}, \ldots, e_{m-1}\right)\right)\right)=\operatorname{Gin}\left(J_{\Gamma_{2}}+\left(e_{2}, \ldots, e_{m-1}\right)\right)$. Let $\tilde{\varphi} \in G L_{n}(K)$ be an automorphism of $E$ defined by $\tilde{\varphi}\left(e_{1}\right)=e_{1}$ and $\tilde{\varphi}\left(e_{k}\right)=\varphi\left(e_{k}\right)$ for $k=2,3, \ldots, n$. Then we have

$$
J_{\Delta_{\tilde{\varphi}}(\Sigma)}=\operatorname{in}\left(\tilde{\varphi}\left(J_{\Sigma}\right)\right)=\operatorname{Gin}\left(J_{\Gamma_{1}}\right) \bigoplus e_{1} \wedge \operatorname{Gin}\left(J_{\Gamma_{2}}+\left(e_{2}, \ldots, e_{m-1}\right)\right) .
$$

Then it follows from Lemmas 2.1 and 3.4 that

$$
\operatorname{Gin}\left(J_{\Gamma_{2}}+\left(e_{2}, \ldots, e_{m-1}\right)\right)=J_{\{n\} * \Delta^{e}\left(\mathrm{lk}_{\Gamma}(i) \cap \mathrm{k}_{\Gamma}(j)\right)}+\left(e_{2}, \ldots, e_{m-1}\right) .
$$

Thus we have

$$
\begin{aligned}
\Delta_{\tilde{\varphi}}(\Sigma) & =\Delta^{e}\left(\Gamma_{1}\right) \cup\left\{\{1\} \cup F: F \in\{n\} * \Delta^{e}\left(\operatorname{lk}_{\Gamma}(i) \cap \operatorname{lk}_{\Gamma}(j)\right)\right\} \\
& \subset \Delta^{e}\left(\Gamma_{1}\right) \cup\left(\{1, n\} * \Delta^{e}\left(\operatorname{lk}_{\Gamma}(i) \cap \operatorname{lk}_{\Gamma}(j)\right)\right) .
\end{aligned}
$$

Since $\Delta^{e}\left(\Gamma_{1}\right) \subset \Delta([2, n], d)$ and $\Delta^{e}\left(\operatorname{lk}_{\Gamma}(i) \cap \mathrm{lk}_{\Gamma}(j)\right) \subset \Delta([m, n-1], d-2)$ by the assumption, Lemma 3.5 implies $\Delta_{\tilde{\varphi}}(\Sigma) \subset \Delta(n, d)$. Then by $\left(S_{2}\right)$ and $\left(S_{4}\right)$ we have

$$
\Delta^{e}\left(\Delta_{\tilde{\varphi}}(\Sigma)\right) \subset \Delta^{e}(\Delta(n, d))=\Delta(n, d) .
$$

Hence we have $\Delta^{e}(\Sigma) \subset \Delta(n, d)$ by Lemma 3.2 as desired.

If $\Gamma$ is the boundary of a simplex then we have $\Delta^{e}(\Gamma)=\Gamma=\Delta(d+1, d)$. Then the next statement follows from Lemma 1.3 and Proposition 3.6 inductively.

Theorem 3.7 If $\Gamma$ is a $(d-1)$-dimensional strongly edge decomposable complex on $[n]$ then $\Delta^{e}(\Gamma) \subset$ $\Delta(n, d)$. 


\section{The strong Lefschetz property}

In this section, we recall the relation between algebraic shifting and the strong Lefschetz property. Let $S=K\left[x_{1}, \ldots, x_{n}\right]$ be a polynomial ring over an infinite field $K$ with each $\operatorname{deg} x_{i}=1$. For a graded $S$-module $M$, we write $M_{k}$ for the homogeneous component of degree $k$ of $M$. We refer the reader to Stanley (1996) for foundations on commutative algebra, such as the Cohen-Macaulay property and linear systems of parameters (1.s.o.p. for short).

Let $\Gamma$ be a $(d-1)$-dimensional simplicial complex on $[n]$. The ring $K[\Gamma]=S / I_{\Gamma}$ is called the Stanley-Reisner ring of $\Gamma$. It is known that the Krull dimension of $K[\Gamma]$ is equal to $d$ (see $\operatorname{Stanley}(1996)$ ). We say that $\Gamma$ has the strong Lefschetz property if $K[\Gamma]$ is Cohen-Macaulay and there exist an 1.s.o.p. $\theta_{1}, \ldots, \theta_{d} \in S_{1}$ of $K[\Gamma]$ and a linear form $\omega \in S_{1}$ such that the multiplication map

$$
\omega^{d-2 i}:\left(K[\Gamma] /\left(\theta_{1}, \ldots, \theta_{d}\right) K[\Gamma]\right)_{i} \rightarrow\left(K[\Gamma] /\left(\theta_{1}, \ldots, \theta_{d}\right) K[\Gamma]\right)_{d-i}
$$

is bijective for $i=0,1, \ldots,\left\lfloor\frac{d}{2}\right\rfloor$. The strong Lefschetz property was used by Stanley $(1980)$ to prove the necessity of the characterization of face vectors of simplicial polytopes (see Stanley (1996) for further information). It was conjectured that all simplicial spheres have the strong Lefschetz property since it yields the characterization of face vectors of simplicial spheres. Conjecture 1 is closely related to this conjecture. Indeed, Kalai (1991) proved the following result.

Lemma 4.1 (Kalai) Let $\Gamma$ be a $(d-1)$-dimensional Cohen-Macaulay complex on $[n]$. Then $\Gamma$ has the strong Lefschetz property in characteristic 0 if and only if $\Delta^{s}(\Gamma) \subset \Delta^{s}(C(n, d))$ and $h_{i}(\Gamma)=h_{d-i}(\Gamma)$ for $i=0,1, \ldots, d$.

On the other hand, strongly edge decomposable complexes are Cohen-Macaulay and have the strong Lefschetz property by the next theorem.

Theorem 4.2 Let $\Gamma$ be a $(d-1)$-dimensional simplicial complex on $[n]$ satisfying the Link condition with respect to $\{i, j\} \subset[n]$. Suppose $\operatorname{dim} \mathcal{C}_{\Gamma}(i j)=d-1$ and $\operatorname{dim} \operatorname{lk}_{\Gamma}(i j)=d-3$.

(i) if $\mathcal{C}_{\Gamma}(i j)$ and $\mathrm{lk}_{\Gamma}(i j)$ have the strong Lefschetz property then $\Gamma$ has the strong Lefschetz property.

(ii) if $\mathcal{C}_{\Gamma}(i j)$ and $\operatorname{lk}_{\Gamma}(i j)$ are Cohen-Macaulay then $\Gamma$ is Cohen-Macaulay.

Theorem 4.2 (i) was first proved by Babson and Nevo (2008) in characteristic 0 (while they did not claim that they proved it, all essential ingredients for the proof appeared in the paper), and the proof in arbitrary characteristic was later given in Murai (2008).

Let $\Gamma$ be a $(d-1)$-dimensional strongly edge decomposable complex on $[n]$. By Theorem $4.2 \Gamma$ is Cohen-Macaulay in arbitrary characteristic. Thus $\Delta^{e}(\Gamma)$ and $\Delta^{s}(\Gamma)$ are pure by Lemma 2.2 Also, the $h$-vector of $\Gamma$ is symmetric by Lemma 1.4 . Moreover, by Theorems 3.7 and 4.2 together with Lemmas 3.1 and 4.1 it follows that $\Delta^{e}(\Gamma) \subset \Delta^{s}(C(n, d))$ and $\Delta^{s}(\Gamma) \subset \Delta^{s}(C(n, d))$. Thus algebraic shifted complexes of strongly edge decomposable complexes satisfy condition (a), (b) and (c) in the Introduction. To summarise, we have

Theorem 4.3 Let $\Delta(-)$ be either $\Delta^{e}(-)$ or $\Delta^{s}(-)$ and let $\Gamma$ be a $(d-1)$-dimensional strongly edge decomposable complex on $[n]$. Then $\Delta(\Gamma)$ is pure, $h_{i}(\Delta(\Gamma))=h_{d-i}(\Delta(\Gamma))$ for $d=0,1, \ldots, d$ and $\Delta(\Gamma) \subset \Delta^{s}(C(n, d))$.

In the next section, we will show that the above theorem characterizes algebraic shifted complexes of strongly edge decomposable complexes. 


\section{$5 \quad$ Algebraic shifting and squeezed spheres}

Squeezed spheres were introduced by Kalai (1988) by extending the construction of Billera-Lee polytopes (Billera and Lee(1981)). The aim of this section is to prove the following result.

Theorem 5.1 Let $\Delta(-)$ be either $\Delta^{e}(-)$ or $\Delta^{s}(-)$ and let $\Sigma$ be a $(d-1)$-dimensional pure shifted simplicial complex on $[n]$ satisfying $h_{i}(\Sigma)=h_{d-i}(\Sigma)$ for $i=0,1, \ldots, d$ and $\Sigma \subset \Delta^{s}(C(n, d))$. Then there exists a squeezed sphere $\Gamma$ such that $\Delta(\Gamma)=\Sigma$.

First we introduce squeezed spheres following (Kalai, 1988, §5.2) and (Murai, 2007b, §2). Fix integers $n>d>0$ and let $m=n-d-1$. Let $\mathcal{M}_{[m]}$ be the set of monomials in $K\left[x_{1}, \ldots, x_{m}\right]$ where $\mathcal{M}_{[0]}=\{1\}$. A set $U$ of monomials in $\mathcal{M}_{[m]}$ is called an order ideal of monomials on $[\mathrm{m}]$ if $U$ satisfies (i) $\left\{1, x_{1}, \ldots, x_{m}\right\} \subset U$ and (ii) if $u \in U$ and $v \in \mathcal{M}_{[m]}$ divides $u$ then $v \in U$. An order ideal $U$ of monomials on $[m]$ is said to be shifted if $u x_{i} \in U$ and $i<j \leq m$ imply $u x_{j} \in U$. For any $u=x_{i_{1}} \cdots x_{i_{k}} \in \mathcal{M}_{[m]}$ with $i_{1} \leq \cdots \leq i_{k}$ and with $k \leq \frac{d+1}{2}$, define a $(d+1)$-subset $F_{d}(u) \subset[n]$ by

$$
F_{d}(u)=\left\{i_{1}, i_{1}+1\right\} \cup\left\{i_{2}+2, i_{2}+3\right\} \cup \ldots \cup\left\{i_{k}+2(k-1), i_{k}+2 k-1\right\} \cup[n+2 k-d, n]
$$

where $F_{d}(1)=[n-d, n]$. Let $U$ be a shifted order ideal of monomials of degree at most $\frac{d+1}{2}$ on $[m]$ and let $B_{d}(U)$ be the simplicial complex generated by $\left\{F_{d}(u): u \in U\right\}$. Kalai (1988) proved that $B_{d}(U)$ is a shellable $d$-ball with $n$ vertices. This simplicial complex $B_{d}(U)$ is called the squeezed d-ball w.r.t. $U$, and its boundary $S_{d}(U)=\partial\left(B_{d}(U)\right)$ is called the squeezed $(d-1)$-sphere w.r.t. $U$. The following fact is known (see (Kalai, 1988, Proposition 5.3)).

Lemma 5.2 (Kalai) Let $d \geq 0$ be an integer and $U$ a shifted order ideal of monomials of degree at most $\frac{d+1}{2}$. Then

$$
\left\{F \in B_{d}(U):|F| \leq \frac{d}{2}\right\}=\left\{F \in S_{d}(U):|F| \leq \frac{d}{2}\right\} .
$$

Simplicial spheres are not always strongly edge decomposable (see (Dey et al. 1999. §7)). However, a class of strongly edge decomposable complexes contains squeezed spheres.

Proposition 5.3 Squeezed spheres are strongly edge decomposable.

Since squeezed spheres are not always polytopal (Kalai (1988)), Proposition 5.3 shows that the class of strongly edge decomposable spheres is not contained in the class of boundary complexes of simplicial polytopes, which is a famous class of simplicial complexes whose Stanley-Reisner ring have the strong Lefschetz property. The proof of Proposition 5.3 is given inductively by using the next lemma.

Lemma 5.4 Let $d>2$ and $n>d+1$ be integers. Let $S_{d}(U)$ be a squeezed sphere on $[n]$. Then $S_{d}(U)$ satisfies the Link condition with respect to $\{1,2\}$ and both $\mathcal{C}_{S_{d}(U)}(\{1,2\})$ and $\operatorname{lk}_{S_{d}(U)}(\{1,2\})$ are combinatorially isomorphic to some squeezed spheres.

Here we omit the proof of Lemma 5.4. (The proof is not difficult, however, we need to check which subsets are facets of $S_{d}(U)$ carefully.)

Next, we will show Theorem 5.1. For a $(d-1)$-dimensional simplicial complex $\Gamma$ on $[n]$, let

$$
U(\Gamma)=\left\{u \in \mathcal{M}_{[n-d-1]}: u \notin \operatorname{Gin}\left(I_{\Gamma}\right)\right\},
$$

where $I_{\Gamma} \subset S$ is the Stanley-Reisner ideal of $\Gamma$ and Gin(-) is the generic initial ideal in characteristic 0 . Kalai (1991) proved the following result (see also (Kalai, 2002, p. 144)). 
Lemma 5.5 (Kalai) Let $\Gamma$ be a $(d-1)$-dimensional simplicial complex on $[n]$ having the strong Lefschetz property in characteristic 0 . Then $U(\Gamma)$ is a shifted order ideal of monomials of degree at most $\frac{d}{2}$ on $[n-d-1]$. Moreover, if $\Sigma$ is a $(d-1)$-dimensional simplicial complex on $[n]$ having the strong Lefschetz property in characteristic 0 and satisfying $U(\Sigma)=U(\Gamma)$ then $\Delta^{s}(\Sigma)=\Delta^{s}(\Gamma)$.

The following result was shown in (Murai, 2007b, Proposition 4.1, Theorem 4.2 and Corollary 7.7).

Lemma 5.6 Let $d \geq 0$ be an integer and $U$ a shifted order ideal of monomials of degree at most $\frac{d+1}{2}$. Then $\Delta^{e}\left(B_{d}(U)\right)=\Delta^{s}\left(S_{d}(U)\right)$. Moreover, if $\max \{\operatorname{deg} u: u \in U\} \leq \frac{d}{2}$ then $U\left(S_{d}(U)\right)=U$.

Proposition 5.7 For any squeezed $(d-1)$-sphere $S_{d}(U)$ one has $\Delta^{e}\left(S_{d}(U)\right)=\Delta^{s}\left(S_{d}(U)\right)$.

Proof: By $\left(S_{2}\right)$ it is enough to show $\Delta^{s}\left(\Delta^{e}\left(S_{d}(U)\right)\right)=\Delta^{s}\left(\Delta^{s}\left(S_{d}(U)\right)\right)$. Note that $\Delta^{e}\left(S_{d}(U)\right)$ and $\Delta^{s}\left(S_{d}(U)\right)$ are Cohen-Macaulay by Lemma 2.2. Since squeezed spheres are strongly edge decomposable, by using $\left(S_{2}\right)$, it follows from Lemma 4.1 and Theorem 4.3 that $\Delta^{e}\left(S_{d}(U)\right)$ and $\Delta^{s}\left(S_{d}(U)\right)$ have the strong Lefschetz property in characteristic 0 . Then, by Lemma 5.5, what we must prove is

$$
U\left(\Delta^{e}\left(S_{d}(U)\right)\right)=U\left(\Delta^{s}\left(S_{d}(U)\right)\right)
$$

Since $U\left(\Delta^{e}\left(S_{d}(U)\right)\right)$ and $U\left(\Delta^{s}\left(S_{d}(U)\right)\right)$ are sets of monomials of degree at most $\frac{d}{2}$ by Lemma 5.5 , it follows from Lemmas 5.2 and 5.6 that

$$
\begin{aligned}
U\left(\Delta^{e}\left(S_{d}(U)\right)\right) & =\left\{u \in \mathcal{M}_{[n-d-1]}: u \notin \operatorname{Gin}\left(I_{\Delta^{e}\left(S_{d}(U)\right)}\right)\right\} \\
& =\left\{u \in \mathcal{M}_{[n-d-1]}: u \notin \operatorname{Gin}\left(I_{\Delta^{e}\left(B_{d}(U)\right)}\right), \operatorname{deg} u \leq \frac{d}{2}\right\} \\
& =\left\{u \in \mathcal{M}_{[n-d-1]}: u \notin \operatorname{Gin}\left(I_{\Delta^{s}\left(B_{d}(U)\right)}\right), \operatorname{deg} u \leq \frac{d}{2}\right\} \\
& =\left\{u \in \mathcal{M}_{[n-d-1]}: u \notin \operatorname{Gin}\left(I_{\Delta^{s}\left(S_{d}(U)\right)}\right)\right\}=U\left(\Delta^{s}\left(S_{d}(U)\right)\right),
\end{aligned}
$$

as desired.

Proof of Theorem 5.1: By Proposition 5.7, it is enough to prove the statement for symmetric algebraic shifting. Since $\Sigma$ is shifted and pure, $\Sigma$ is Cohen-Macaulay by Lemma 2.2. Also, since $\Delta^{s}(\Sigma)=\Sigma$ by $\left(S_{2}\right)$, the assumption and Lemma 4.1 imply that $\Sigma$ has the strong Lefschetz property in characteristic 0 . Then by Lemma 5.5 it follows that $U(\Sigma)$ is a shifted order ideal of monomials of degree at most $\frac{d}{2}$.

We will show $\Delta^{s}\left(S_{d}(U(\Sigma))\right)=\Delta^{s}(\Sigma)=\Sigma$. Since squeezed spheres have the strong Lefschetz property by Theorem 4.2 and Proposition 5.3 , what we must prove is $U\left(S_{d}(U(\Sigma))\right)=U(\Sigma)$. However, this equation immediately follows from Lemma 5.6

\section{References}

Babson, E. and Nevo, E. (2008). Lefschetz properties and basic constructions on simplicial spheres. arXiv:0802.1058, preprint.

Babson, E., Novik, I. and Thomas, R. (2006). Reverse lexicographic and lexicographic shifting. J. Algebraic Combin. 23, 107-123. 
Billera, L.J. and Lee, C.W. (1981). A proof of the sufficiency of McMullen's conditions for $f$-vectors of simplicial convex polytopes. J. Combin. Theory Ser. A 31, 237-255.

Björner, A. and Kalai, G. (1989). On $f$-vectors and homology. in: G. Bloom, R. Graham and J. Malkevitch (Eds), Combinatorial Mathematics: Proc. 3rd Intern. Conf., New York, 1985, Annals of N. Y. Acad. Sci., vol. 555, New York Acad. Sci., pp. 63-80.

Dey, T.K., Edelsbrunner, H., Guha, S. and Nekhayev, D.V. (1999). Topology preserving edge contraction. Publ. Inst. Math. (Beograd) (N.S.) 66(80), 23-45.

Eisenbud, D. (1995). Commutative algebra with a view toward algebraic geometry. Grad. Texts in Math., vol. 150, Springer-Verlag, New York.

Erdös, P., Ko, C. and Rado, R. (1961). Intersection theorems for systems of finite sets. Quart. J. Math. Oxford Ser. (2) 12, 313-320.

Herzog, J. (2002). Generic initial ideals and graded Betti numbers. in: T. Hibi (Ed.), Computational commutative algebra and combinatorics. Adv. Stud. Pure Math., vol. 33, Math. Soc. Japan, Tokyo, pp. $75-120$.

Kalai, G. (1984). A characterization of $f$-vectors of families of convex sets in $\mathbb{R}^{d}$. Part I: Necessity of Eckhoff's conditions. Israel J. Math. 48, 175-195.

Kalai, G. (1988). Many triangulated spheres. Discrete Comput. Geom. 3, 1-14.

Kalai, G. (1991), The diameter of graphs of convex polytopes and $f$-vector theory. in: P. Gritzmann and B. Sturmfels (Eds.), Applied geometry and discrete mathematics. DIMACS Ser. Discrete Math. Theoret. Comput. Sci., vol. 4, Amer. Math. Soc., Providence, pp. 387-411.

Kalai, G. (2002). Algebraic shifting. in: T. Hibi (Ed.), Computational commutative algebra and combinatorics. Adv. Stud. Pure Math., vol. 33, Math. Soc. Japan, Tokyo, pp. 121-163.

Murai, S. (2007a). Algebraic shifting of cyclic polytopes and stacked polytopes. Discrete Math. 307, $1707-1721$.

Murai, S. (2007b). Generic initial ideals and squeezed spheres. Adv. Math. 214, 701-729.

Murai, S. (2008). Algebraic shifting of strongly edge decomposable spheres. arXiv:0709.4518, preprint.

Murai, S. and Hibi T. (2005). Algebraic shifting and graded Betti numbers. Trans. Amer. Math, Soc., to appear.

Nevo, E. (2005). Algebraic shifting and basic constructions on simplicial complexes. J. Algebraic Combin. 22, 411-433.

Nevo, E. (2006). Higher minors and Van Kampen's obstruction. arXive:math.CO/0602531, Math. Scand., to appear.

Stanley, R.P. (1980). The number of faces of a simplicial convex polytope. Adv. Math. 35, 236-238.

Stanley, R.P. (1996). Combinatorics and commutative algebra. Second edition. Progr. Math., vol. 41, Birkhäuser, Boston. 
